\title{
Research on Evaluating the Operation of Power Supply Companies
}

\author{
Zhongfu Yu*, Shengfan Ye, Shuyuan Tang \\ The Economics and Management Department, North China Electric Power University, Beijing, China
}

\section{Email address:}

yu_zhongfu@163.com (Zhongfu Yu),648970963@qq.com (Shengfan Ye), 869748729@qq.com (Shuyuan Tang)

*Corresponding author

\section{To cite this article:}

Zhongfu Yu, Shengfan Ye, Shuyuan Tang. Research on Evaluating the Operation of Power Supply Companies. Journal of Finance and Accounting. Vol. 4, No. 2, 2016, pp. 58-63. doi: 10.11648/j.jfa.20160402.15

Received: March 29, 2016; Accepted: April 6, 2016; Published: April 15, 2016

\begin{abstract}
Power supply companies undertake the important duties of transmission, distribution, the sales of electricity and other social services in the power system, the quality of whose operating conditions directly affect the sustainable development of the corporations and the normal operation of the entire electricity market. The tailored and comprehensive evaluation system to operating conditions in power supply companies has a practical significance. Considering the important role of Power supply companies plays in the national econony, the paper is to develop a performance evaluation index system and comprehensive evaluation model, hoping to provide references for the future performance evaluation management in the power supply enterprises. Based on the concept of comprehensive value, this paper has constructed the "four-dimensional" comprehensive performance evaluation system combined with the operating characteristics, including the circumstances, the quality of assets, the service level and performance level. Meanwhile, a comprehensive evaluation model is built by the Structural Entropy Weight Method and TOPSIS Method and six power supply companies are taken as sample companies. Empirical analysis results verify the effectiveness of the method. The study proposes a new concept of comprehensive value, develops a new method for performance evaluation of power supply companies.
\end{abstract}

Keywords: Power Supply Companies, Operating Conditions, Comprehensive Performance, TOPSIS Method

\section{Introduction}

As is the fundamental energy industry of national economy, the power industry is a vital guarantee of economic development and social progress. Increasingly, it is becoming a consensus that electricity consumption is even known as the "barometer" of the national economy. During the year 2015, Chinese power system reform has taken some significant steps. The No.9 item in the document makes it clear that the direction of reform is "open the ends, regulate the middle", and the fundamental ideology is to open the power generating side and the selling side, leading into the pattern that the price is well dominated by the market competition while transmission and distribution are the main responsibility of power grid corporation strictly controlled by the government. Certainly, it has broken the previous balance that the grid company has "a dominant" monopoly, which has reduced the profit space of the power companies, causing a great competition pressure on power grid companies, especially the power supply companies.

The operational objective of corporations is to create and maximize the value. In China, the power supply companies are not only independent bodies in the competitive market, but also have a natural characteristic of utilities to perform community service, not only solve the employment problems, conserve the energy and do other social responsibilities, but also achieve the profitable operational goals. It is particularly important to know how to establish a proper comprehensive performance evaluation system in order to perspect the operational conditions of the power supply companies scientifically.

Some literatures focus on the application of Structural Entropy Weight Method and TOPSIS Method in performance evaluation of power supply companies. Zhengyuan Jia constructed an operational performance evaluation model for the regional power supply company using Entropy Weight Method and Fuzzy Comprehensive Evaluation Method [1]. Based on the actual research data, Yuanchao $\mathrm{Hu}$ constructed 
an intelligent evaluation model in transformer substation equipment, and calculated the weight of each evaluation index by using the method combines AHP and Entropy Weight Method, then got the result of comprehensive evaluation by improved TOPSIS Method [2]. By using the Legal Right of Entropy, Qingyou Yan evaluated the economic performance of 5 regional power supply companies in a model which combines TOPSIS and Gray Correlation [3].

As most of literatures related to the power supply company focused on economic performance, and researches assimilate the ideology of comprehensive value evaluation management to the performance evaluation of power supply company have not be collected yet, this paper attempts to construct a relatively scientific and effective comprehensive performance of evaluation system in operational conditions and has established a comprehensive evaluation model of power supply companies for operation status with Structure Entropy Method and Topsis Method, hoping to provide a reference to enhance the capacity of the development of power supply companies.

\section{Comprehensive Performance Evaluation System}

\subsection{Values and Design Principles}

Based on Stakeholder Theory, as a vital part in the whole power system, power supply companies bear important responsibilities in electricity production and consumption respects. Their goals are not only to take into the account of their economic benefits, but also consider the value chain of suppliers in the power system, the interests of consumers and respond to the concerns of relevant governmental departments, the public, enterprise employees, and many other market participants. Nowadays, we can find out that the environmental protection and livelihood themes are becoming more important, those enterprises in "closed doors" only to pursue the economic benefits in spite of environmental pollution, public service and people's livelihood will be eliminated. So, the enterprise's management goal lies in the pursuit of economic value, social value and environmental value maximization, the index system of performance evaluation must also consider these three aspects meanwhile. At the same time, as the majority of the power transmission and distribution, power grid companies have their own unique operating characteristics. The assessment indicators should be investigated into both profits and other traditional performance levels, and be applied to examine the traditional operating performance, safety of the power grid, assets operating efficiency, service levels and other aspects of society.

According to the ideology above, the comprehensive evaluation on the operating conditions of those power supply companies should be focused on three aspect, they are economic performance, social performance and environmental performance, and the evaluation indicators should be designed clearly to reflect four dimensions of operating status, including grid conditions, asset quality, service level and performance level.

(1) Grid conditions refer to whether the network planning is scientific, and whether the grid structure is reasonable, safe, reliable, efficient and environmental friendly. In general, the evaluation indicators includes the N-1 grid pass rate, the capacity-load ratio, proportion of single-line and single change, the contact rate of the urban distribution line and the reliability rate of the power system.

(2) Asset quality refers to whether the asset structure is solid and reasonable, whether the cost of the capital is well controlled, and whether the efficiency of assets is sustainable and efficient. The evaluation indicators typically include total assets, rate of return on common stockholders' equity, debt to assets ratio, etc.

(3) Service level means whether the power supply is safe, economical, clean and sustainable, those universal social services are civilized and efficient. The evaluation indicators typically include the reliability rate of the power supply and the qualification rate of the power supply voltage.

(4) Performance level refers to the operational performance of power grid enterprises in the process of production and operation, the evaluation indexes typically contain electricity sales, the line loss rate, the total profit, the economic value added and so on.

\subsection{Evaluation Index System}

According to the significant degree between the management performance and the indicators, and consider the problems of the internal logic and co-linearity of indicators, we have provided with the comprehensive performance evaluation system, which is shown in Figure 1, by taking a combination of qualitative and quantitative analysis methods and using experts' experience.

\section{Comprehensive Assessment Model on Operating Conditions}

\subsection{Determine the Evaluation of Index Weight Under Structure Entropy Method}

To improve the accuracy of the evaluation results, we use the structure entropy method to determine the weight of each index. Combined with the Delphi and fuzzy analysis method, the Entropy Structure Method has both qualitative and quantitative analysis. We use this method to do "typical sort" in the opinions of expert evaluation and then follow the entropy formula to calculate the entropy value and to do the analysis of the "blind degree", which results to an index weight after completing the normalized process.

Based on the operating data in 2014 of the $\mathrm{A} \sim \mathrm{F}$ six power supply companies belonged to the Power Company in $\mathrm{H}$ province, we have done the comprehensive evaluation on the operating conditions. 
Firstly, we divide 30 experts who are in the part of grid operation analysis into 3 groups evenly to do the typical order [4-5]. The results of the first index in the grid-level business conditions are shown in the Table 1.

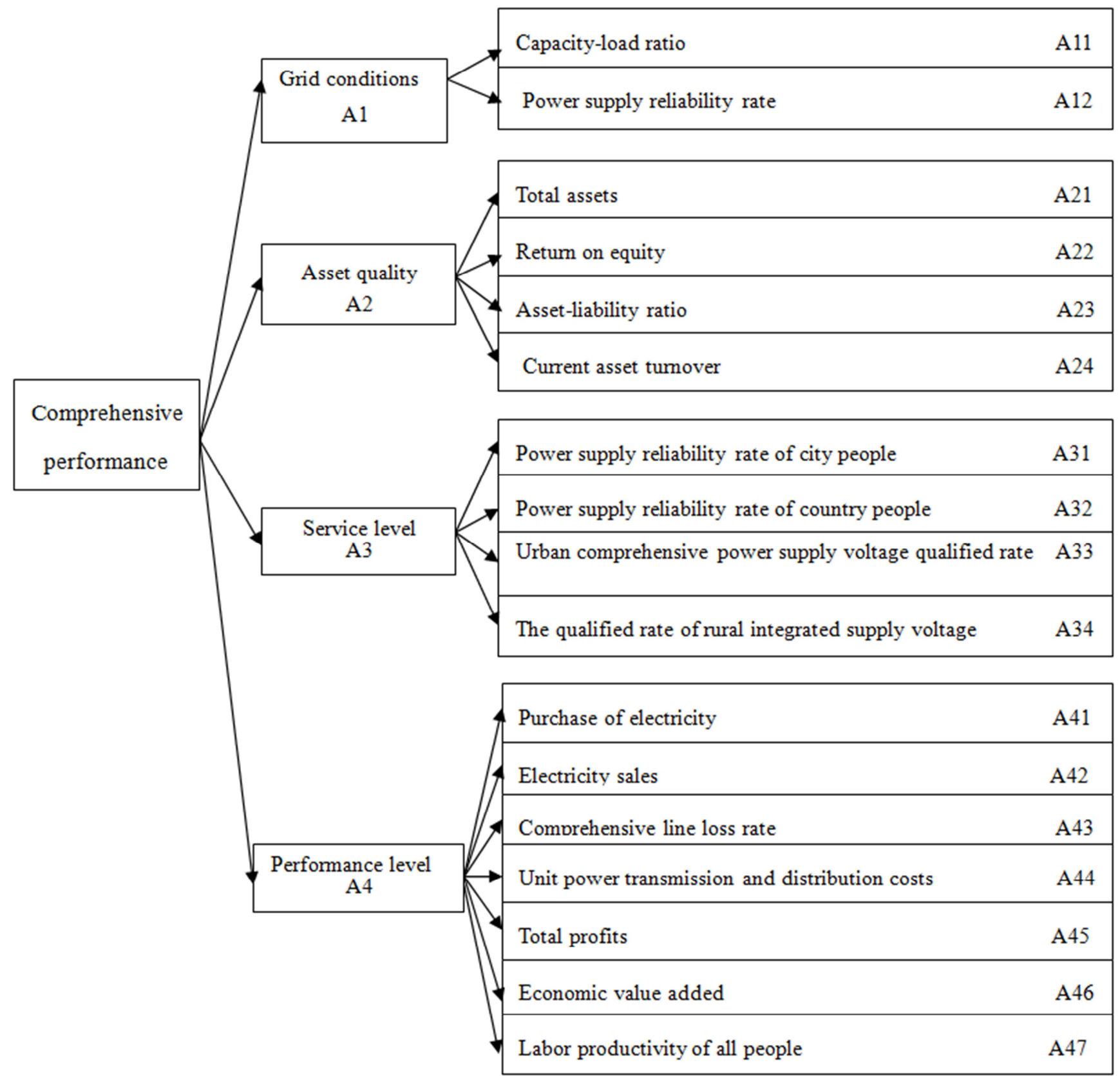

Figure 1. Comprehensive evaluation indicator system.

Table 1. The evaluation indicators of the first floor, $\lambda=5$.

\begin{tabular}{lllll}
\hline Items & Grid conditions & Asset quality & Service level & Performance level \\
\hline first group sorted & 2 & 3 & 4 & 1 \\
second group sorted & 2 & 4 & 3 & 1 \\
third group sorted & 1 & 3 & 4 & 2 \\
Average above & 0.8617 & 0.3333 & 0.1667 & 0.9308 \\
Maximum & 1 & 0.5 & 0.5 & 1 \\
Average above & 0.1383 & 0.1667 & 0.3333 & 0.0692 \\
Minimum & 0.7925 & 0 & 0 & 0.7925 \\
Average above & -0.0692 & -0.3333 & -0.1667 & -0.1383 \\
Cognitive blind degree Q & 0.0346 & 0.0833 & 0.0833 & 0.0346 \\
1-Q & 0.9654 & 0.9167 & 0.9167 & 0.9654 \\
Total assessment & 0.8319 & 0.3056 & 0.1528 & 0.8986 \\
Weight & 0.3800 & 0.1396 & 0.0698 & 0.4106 \\
\hline
\end{tabular}


Then, by calculating, the secondary indicators may be further sorted and the results of asset quality are shown in Table 2 .

Table 2. The evaluation indicators of the second floor(Asset quality), $\lambda=6$.

\begin{tabular}{lllll}
\hline Items & Total assets & Return on equity & Asset-liability ratio & Current asset turnover \\
\hline first group sorted & 4 & 1 & 2 & 3 \\
second group sorted & 3 & 1 & 2 & 4 \\
third group sorted & 4 & 2 & 1 & 3 \\
Average above & 0.5147 & 0.9538 & 0.9076 & 0.5986 \\
Maximum & 0.6826 & 1 & 1 & 0.6826 \\
Average above & 0.1680 & 0.0462 & 0.0924 & 0.0840 \\
Minimum & 0.4307 & 0 & 0.8614 & 0.4307 \\
Average above & -0.084 & -0.9538 & -0.0462 & -0.1680 \\
Cognitive blind degree Q & 0.0420 & 0.4538 & 0.0231 & 0.0420 \\
1-Q & 0.9580 & 0.5462 & 0.9769 & 0.9580 \\
Total assessment & 0.4930 & 0.5210 & 0.8866 & 0.5735 \\
Weight on asset quality & 0.1993 & 0.2106 & 0.3584 & 0.2318 \\
Weight on operations & 0.0278 & 0.0294 & 0.0500 & 0.0324 \\
\hline
\end{tabular}

Finally, after comprehensive calculation, we can conclude to the result of the indicators' weight according to the structure entropy method shown in Table 3.

Table 3. The evaluation index of business conditions of the power supply company.

\begin{tabular}{llll}
\hline Objective level A & Standard level Ai & Index level Aij & The weight from index layer to the target layer Wij \\
\hline & A1 & A11 & 0.2534 \\
& 0.3800 & A12 & 0.1267 \\
& A2 & A21 & 0.0278 \\
& 0.1396 & A23 & 0.0294 \\
& & A24 & 0.0500 \\
Operation Conditions on & A3 & A31 & 0.0324 \\
The Power Grid & A.0698 & A32 & 0.0236 \\
Corporations & & A33 & 0.0113 \\
& & A41 & 0.0225 \\
& & A42 & 0.0125 \\
& A4 & A43 & 0.0532 \\
& 0.4106 & A44 & 0.0590 \\
& & A45 & 0.0713 \\
& & A46 & 0.0411 \\
& A47 & 0.0758 \\
\end{tabular}

\subsection{The Comprehensive Evaluation Based on TOPSIS}

TOPSIS method is sorted according to the close relationship between specific evaluation object and idealized target, which has been widely used in the fields of the risk assessment [6-7], the multi-objective optimization [8-9], the network planning and so on [10-11]. The comprehensive evaluation model which is constructed with the help of the TOPSIS method can analyze and compare the trends how the operating conditions of the power supply company changes in several consecutive period and can also compare and evaluate the same level of operating conditions in a horizontal level of the power supply company to achieve the comprehensive grasp of the power supply company's operating conditions vertically and horizontally.

The comprehensive evaluation of each indicator scores by calculation is followed under the TOPSIS method.

Step one: construct the initial matrix (n objects to be evaluated, $m$ evaluation indexes).

- Evaluation indicators change following the trend, making the value which changes after any value of a property under the excellent performance program is transformed in the standardization of the table greater.

- To be dimensionless, excluding the dimensional effect on the decision or the assessment.

- To do the normalization of the raw data matrix which changes following the trend, and also to construct a corresponding matrix. (Table values are converted to $[0,1]$ interval)

Step Two: construct a weighted standardized matrix.

$$
V_{i j}=\left[\begin{array}{cccc}
\mathrm{w}_{1} y_{11} & \mathrm{w}_{2} y_{12} & \cdots & \mathrm{w}_{\mathrm{m}} y_{1 m} \\
\mathrm{w}_{1} y_{21} & \mathrm{w}_{2} y_{22} & \cdots & \mathrm{w}_{\mathrm{m}} y_{2 m} \\
\vdots & \vdots & & \vdots \\
\mathrm{w}_{1} y_{n 1} & \mathrm{w}_{2} y_{n 2} & \cdots & \mathrm{w}_{\mathrm{m}} y_{n m}
\end{array}\right]=\left[\begin{array}{cccc}
V_{11} & V_{12} & \cdots & V_{1 m} \\
V_{21} & V_{22} & \cdots & V_{2 m} \\
\vdots & \vdots & & \vdots \\
V_{n 1} & V_{n 2} & \cdots & V_{n m}
\end{array}\right]
$$

In the chart, Wi represents the weight of indicators which are determined by structure entropy method.

Step 3: define the positive ideal and negative ideal. 


$$
\begin{aligned}
& V^{+}=\left\{\left(\max V_{i j} \mid j \in J^{+}\right),\left(\min V_{i j} \mid j \in J^{-}\right)\right\} \\
& =\left(V_{1}^{+}, V_{2}^{+}, \cdots, V_{m}^{+}\right), \\
& V^{-}=\left\{\left(\min V_{I J} \mid j \in J^{+}\right),\left(\max V_{i j} \mid j \in J^{-}\right)\right\} \\
& =\left(V_{1}^{-}, V_{2}^{-}, \cdots, V_{m}^{-}\right)
\end{aligned}
$$

$J^{+}$refers to the benefit attribute while $J^{-}$is on behalf of the cost attribute.

Step 4: Calculate the distance between the assessment objectives and the positive or the negative ideal point.

$$
\begin{gathered}
S_{i}^{+}=\sqrt{\sum_{j=1}^{m} W_{j}\left(V_{i j}-V_{j}^{+}\right)^{2}},(i=1,2, \cdots, n) \\
S_{i}^{-}=\sqrt{\sum_{j=1}^{m} W_{j}\left(V_{i j}-V_{j}^{-}\right)^{2}},(i=1,2, \ldots, n)
\end{gathered}
$$

$S^{+}$and $S^{-}$respectively represent the distance between the assessment indicators and the positive or negative ideal point.

Step Five: calculate the line indicated value and rank. C represents the queue indicated value, which reflects the distance between the negative evaluation objects and the negative ideal point. The greater $\mathrm{C}$ is, the farther the distance is and vice versa. Line indicator value is calculated as follows:

$$
C_{i}^{*}=\frac{S_{i}^{-}}{S_{i}^{+}+S_{i}^{-}}, i=1,2, \cdots n
$$

Based on the size of the $\mathrm{C}$ value, the evaluation objects are sorted according to the definition of $\mathrm{C}$, as a result of which the larger $\mathrm{C}$ is, the more excellent the assessment object is and vice versa [12].

According to the index weights calculated in Table 3 as follows:

$\mathrm{Wj}=(0.2534,0.1267,0.0278,0.0294,0.0500,0.0324,0.0236,0$ $.0113,0.0225,0.0125,0.0532,0.0590,0.0713,0.0411,0.0758,0.0$ 788,0.0314)

\begin{tabular}{|c|c|c|c|c|}
\hline \multirow{2}{*}{ Evaluation object } & \multicolumn{2}{|c|}{ The distance from the evaluation object to the desired point } & \multirow{2}{*}{ Queuing indicated value } & \multirow{2}{*}{ Rank } \\
\hline & $\mathbf{S}^{+}$ & $\mathbf{S}^{-}$ & & \\
\hline A & 0.8603742 & 2896937.824902 & 0.9999997 & 1 \\
\hline B & 2424835.7451753 & 474701.0782747 & 0.1637162 & 3 \\
\hline $\mathrm{C}$ & 2704148.1906264 & 203396.8868191 & 0.0699549 & 4 \\
\hline $\mathrm{D}$ & 2866043.7398165 & 31308.1548377 & 0.0108058 & 5 \\
\hline $\mathrm{E}$ & 2896740.6987811 & 10220.7952895 & 0.0035160 & 6 \\
\hline $\mathrm{F}$ & 1516935.9288657 & 1660662.6872026 & 0.5226156 & 2 \\
\hline
\end{tabular}

By calculation, based on the ideal point method, the comprehensive evaluation of the operational conditions in 2014 of the six municipal power supply companies which are pertained to H Provincial Power Company is shown in the Table 4.

Table 4. Rank of the operation status in the power supply company.

According to Table 4, based on the results of the queue indicated value, we can find out that the line indicator of the power supply company in A city (county) gains the highest value, which indicates that the distance between the company and the negative ideal is the farthest and the consolidated operating performance should be the best.

Similarly, using the model above, a company can also be focused on as the object of assessment. In the comprehensive analysis of the change in operation performance fluctuations of the corporation for many years constantly, we can examine the influence of the implication using the results of evaluation tests. However, due to the constraints of the space, we can only give the evaluation results of operating conditions on which several companies have operated in the same period.

\section{Conclusion}

At the time of a new round of power to change, the grid enterprises, especially for the power supply companies, will face a more severe market competition. How to evaluate the operation conditions scientifically and enhance the corporation value by internal potential is a vital proposition under the new situation. By integrating the Structural Entropy and the Ideal Point method, we have established a comprehensive evaluation model based on the integrated value-oriented model of operation status of power supply companies, which can achieve the comparison of the operating conditions of those power supply companies horizontally and longitudinally in different parts and years to be more objective to do effectiveness and efficiency of business operations on the grid power.

Because of the availability of the basic data and the uncertainty of evaluation criteria by regulatory authorities policy, the comprehensive evaluation model which is established in this paper still requires power supply companies take full account of policy factors and score to each index realistically when in a practical application process, and try to ensure the reliability of evaluation results in comprehensive evaluation on operating status, which is the disadvantage and the improvement of various evaluation methods.

\section{References}

[1] Jia Zhengyuan, Wang Chunmei, Huang Zhiwei, Zhang Gang. Evaluation Research of Regional Power Grid Companies' Operation Capacity based on Entropy Weight Fuzzy Comprehensive Model. Procedia Engineering 2011 (15): 46264630 . 
[2] Yuanchao Hu, Ling Ruan, Jiangjun Ruan, Qijia Xie, Tao Huang, Zhizheng Li, Evaluation on Intelligent Renovation of Substations Based on Improved TOPSIS, Power System Technology, 10: 42-48, 2012.

[3] Qingyou Yan, Zhouyang Li, Assessment on Economic Evaluation of Smart Grid Based on TOPSIS Method and Grey Correlation Degree Analysis, 02: 179-184, 2016.

[4] Qiyue Cheng, The structure entropy method to evaluate index weights, the theory and practice for engineering systems, 1225-1229, 30 (7) (2010).

[5] Ying Zeng, Wenhong Zhu, The evaluation method of the reliability of the ICT communication network based on the law of entropy power structure, East China Electric Power, 0097-0100, 42 (1) (2014).

[6] Yanbin Li, Xinyi Yu, Zhijie Wang, The risky evaluation research on the power generation project with the help of the gray correlation and TOPSISI method, Power System Technology, 1514-1519, 37(6) (2013).

[7] Xianchang Cao, Jinyuan Shi, Anzong Jiang, etc, The quantitative and risky research on the error of the power station equipment based on the fuzzy mathematics, Proceedings of the csee, 93-100, 25(23) (2005).

[8] Xuebin Li, The multi-objective and decision research on the distribution of the overloading of the power plant, Proceedings of the csee, 102-107, 28(35) (2008).

[9] Jiekang $\mathrm{Wu}, \mathrm{Li}$ Tang, The multi-objective stochastic scheduling model for the hydrothermal power systems based on the interactive fuzzy chance constrained programming, Proceedings of the csee, 26-34, 31(25) (2011).

[10] Qian Wang, Lizi Zhang, The multi-objective network planning of the wind power plant using the hybrid intelligent algorithm of NSGA-II, Proceedings of the csee, 17-24, 31(19) (2011).

[11] Xinran Li, Yanghua Liu, Xiangyou Zhu, etc, The evaluation index system and its application on the grid construction scale, Proceedings of the csee, 17-24, 26(17) (2006).

[12] Yong Xiang, Hong Ran, The evaluation studies on smart city based on ANP-TOPSIS method, The Industrial technology economics, 131- 136, 04(2014). 